\title{
Gesundheit ermöglichen
}

\section{Das Konzept der Salutogenese}

\author{
Ursula Sottong
}

Dr. med. Ursula Sottong ist Ärztin und Gesundheitswissenschaftlerin. Sie leitet die Malteser Gesundheitsförderung und Prävention und die Malteser Akademie in Köln. Die Malteser Akademie ist die bundesweite Entwicklungs-,

Koordinations- und Controllingstelle für alle zentralen Bildungsangebote der Malteser.

Internet http://www.malteser.de

E-Mail Ursula.Sottong@malteser.de
Gesundheit kann heute nicht mehr als bloße Abwesenheit von Krankheit verstanden werden. Insbesondere das Konzept der Salutogenese geht davon aus, dass Gesundheit nicht als Zustand, sondern als Prozess zu verstehen ist, der durch den Menschen selbst, aber auch durch seine Umwelt beeinflusst werden kann.

Gesundheit wurde im Jahre 1948 in der Konstitution der Weltgesundheitsorganisation (WHO) als "Zustand völligen körperlichen, seelischen und sozialen Wohlbefindens " und nicht nur als das "Freisein von Krankheit und Gebrechen" beschrieben.

Eine historische Wende im Gesundheitsverständnis wurde im November 1986 mit der Verabschiedung der Ottawa-Charta eingeleitet: »Gesundheitsförderung zielt auf einen Prozess, allen Menschen ein höheres Maß an Selbstbestimmung über ihre Gesundheit zu ermöglichen und sie damit zur Stärkung ihrer Gesundheit zu befähigen «. Die Gedanken der Ottawa-Charta basieren auf dem Prinzip der Salutogenese. Seither geht es der WHO unter dem Motto »Gesundheit für alle « darum, jene Bedingungen und Ressourcen zu stärken, die Menschen gesund erhalten. Diese neue Sichtweise hat sich unter dem Namen »Gesundheitsförderung « etabliert.

Das Konzept der Salutogenese (salus = Unversehrtheit; genese = Entstehung) geht auf den amerikanisch-israelischen Soziologen Aaron Antonovsky (19231994) zurück. Er fragt nicht nach den Ursachen von Erkrankungen (»Warum werden Menschen krank? «), sondern nach dem, was Menschen gesund erhält. Damit löst er sich vom klassischen medizinischen Verständnis, das pathogenetisch geprägt ist und nach der Entstehung und den Bedingungen von Krankheit fragt.

Aron Antonovsky wertete im Jahre 1970 eine Erhebung über die Adaption von Frauen verschiedener ethnischer Gruppen an die Menopause aus. Eine Gruppe von ihnen war 1939 zwischen 16 und 25 Jahren alt gewesen und hatte sich zu dieser Zeit in einem national-sozialistischen Konzentrationslager befunden. Ihre emotionale Befindlichkeit wurde mit der einer Kontrollgruppe verglichen. Der Anteil der in ihrer Gesundheit beeinträchtigten Frauen betrug in der Kontrollgruppe 51 Prozent, im Vergleich zu 71 Prozent der KZ-Überlebenden. Nicht der Unterschied an sich, sondern die Tatsache, dass in der Gruppe der KZ-Überlebenden 29 Prozent der Frauen trotz der unvorstellbaren Qualen eines Lagerlebens mit anschließendem Flüchtlingsdasein als körperlich und psychisch 'gesund beurteilt wurden, war für ihn ein unerwartetes Ergebnis. Diese Beobachtung führte ihn zu der Frage, welche Eigenschaften und Ressourcen diesen Menschen geholfen hatten, unter den Bedingungen der KZHaft sowie in den Jahren danach ihre (körperliche und psychische) Gesundheit zu erhalten. So schuf Antonovsky (im Gegensatz zum Pathogenesekonzept der traditionellen Medizin) das Konzept der `Salutogenese $<$ - der Entstehung von Gesundheit. «(1)

Antonovsky geht in seinen Betrachtungen davon aus, dass Gesundheit »kein normaler, passiver Gleichgewichtszustand « (Homöostase) ist, sondern ein »labiles, aktives und sich dynamisch regulierendes Geschehen « (Heterostase). Gesundheit und Krankheit sind damit Teil eines kontinuierlichen Prozesses, an dessen Endpolen völlige Gesundheit bzw. Krankheit stehen. Der Mensch bewegt sich auf diesem Kontinuum, erreicht aber nie einen der beiden Extrempole, ist also nie nur krank oder nur gesund.

Das klassische Bild, das Antonovsky für diese Dynamik benutzt, ist der Mensch, der sich in einem Fluss bewegt. Er muss dabei Stromschnellen, Wasserfälle und Untiefen überwinden, kommt gelegentlich auch in ruhigere Zonen und 
überlebt die Gefahrenzonen letztlich nur, wenn ihm ausreichend persönliche (Widerstands-) Ressourcen und Schutzfaktoren zur Verfügung stehen und eine
Lebenslagen, gesundheitlicher Beeinträchtigungen, Armut, Traumata etc. ergeben, "gesund « bleiben oder werden. Damit gibt er dem Individuum, aber auch

\section{»Gesund bleibt, wer glaubt, die Kontrolle über sein Leben zu haben «}

entsprechende Grundeinstellung (Überlebenswillen) vorhanden ist.

Antonovsky nennt diese persönliche Ausstattung »Kohärenzgefühl « (sense of coherence). Das ist aber nichts anderes als die Fähigkeit eines Menschen, die Welt als zusammenhängend und sinnvoll zu erleben und seine vorhandenen Ressourcen so zu nutzen, dass sie zu seiner Gesundheit und zu seinem Wohlgefühl beitragen.

Die Ausprägung und Stärke dieses »sense of coherence « hängt von der Lebenserfahrung eines Menschen $a b$, von der Gesellschaft, in der er lebt, und der sozialen Rolle, die der Mensch übernimmt. Dieses Kohärenzgefühl wirkt aber auch auf den Umgang mit neuen Lebenserfahrungen zurück. Antonovsky beschreibt die drei Komponenten des Kohärenzgefühls wie folgt:

Erstens: Gefühl der Verstehbarkeit (sense of comprehensibility), also die Fähigkeit, die Umwelt so zu ordnen und zu strukturieren, dass sie sinnvoll interpretiert werden kann.

Zweitens: Gefühl der Machbarkeit (sense of manageability), also der Glaube und die Überzeugung, Situationen und Probleme aktiv bewältigen zu können.

Drittens: Gefühl der Sinnhaftigkeit (sense of meaningfullness), also etwas bewältigen zu wollen, weil es Sinn macht oder einen Sinn hat, einen Sinn gibt.

Für die Ausprägung des Kohärenzgefühls müssen alle drei Aspekte vorhanden sein, wobei dem Aspekt der Sinnhaftigkeit die größte Bedeutung zukommt.

Was bedeutet das für die Gesundheit? Antonovsky stellt die Frage, warum Menschen gesund bleiben und zeigt auf, wie auf Grund und welcher »heilsamer Ressourcen « Menschen trotz Risikofaktoren, die sich aus dem alltäglichen Leben, belastenden Situationen, schwierigen den Helfenden Aspekte an die Hand, mit welchen Faktoren sie »Heilungsprozesse « oder »Gesunderhaltungsprozesse « unterstützen können:

\section{Was man heute unter «Gesundheit« versteht}

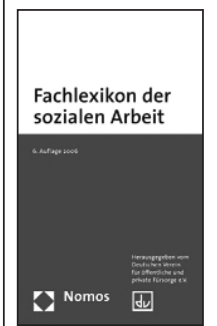

Da Gesundheitsvorstellungen soziokulturellen und historischen Einflüssen und Veränderungen unterliegen und Gesundheit in verschiedenen Epochen, Kulturen und innerhalb einer Gesellschaft unterschiedlich wahrgenommen und verstanden wurde und wird, ist es nicht möglich, ein allgemeines, universales Substrat von Gesundheit zu finden. Folglich kann es auch keine allgemeingültige Definition des Begriffs Gesundheit geben. Gesundheit ist ein relationaler Begriff, der erst im Bezugskontext praktische Bedeutung gewinnt. Entsprechend den vielfältigen Vorstellungen, was Gesundheit bedeutet, differieren auch die zahlreichen (wissenschaftlichen) Versuche, den Begriff zu bestimmen. [...]

Mit dem Ziel, zu einer dem heutigen theoretischen Entwicklungsstand angemessenen und zugleich interdisziplinär verwendbaren Arbeitsdefinition von Gesundheit zu gelangen, hat Hurrelmann (Gesundheitssoziologie, 6. Auflage, Juventa Verlag, Weinheim und München 2006) die Grundvorstellungen zentraler Theorien von Gesundheit und Krankheit überprüft und dabei vier Leitvorstellungen von Gesundheit und Krankheit heraus gearbeitet:

(1) die Leitvorstellung von Gesundheit als gelungener und Krankheit als nicht gelungener Bewältigung von inneren und äußeren Anforderungen,

(2) die Leitvorstellung von Gesundheit als Gleichgewicht und Krankheit als Ungleichgewicht von Risiko- und Schutzfaktoren auf der körperlichen, psychischen und sozialen Ebene,

(3) die Leitvorstellung von »relativer « Gesundheit und »relativer « Krankheit nach objektiven und subjektiven Kriterien,

(4) die Leitvorstellung von Gesundheit und Krankheit als Reaktion auf gesellschaftliche Gegebenheiten.

Diese Leitvorstellungen hat Hurrelmann in folgendem Vorschlag einer konsensuellen Definition von Gesundheit zu verbinden gesucht: »Gesundheit ist das Stadium des Gleichgewichts von Risikofaktoren und Schutzfaktoren, das eintritt, wenn einem Menschen eine Bewältigung sowohl der inneren (körperlichen und psychischen) als auch äußeren (sozialen und materiellen) Anforderungen gelingt. Gesundheit ist ein Stadium, das einem Menschen Wohlbefinden und Lebensfreude vermittelt. «

Gesundheit ist damit eine lebensgeschichtlich immer wieder neu und aktiv herzustellende Balance.

Karl Kälble

Quelle: Deutscher Verein für öffentliche und private Fürsorge e. V. (Hg.): Fachlexikon der sozialen Arbeit. 6. Auflage. Nomos Verlagsgesellschaft, Baden-Baden 2007. 1.195 Seiten. 44,- Euro. ISBN 978-3-8329-1825-5. Seite $404 f$. 